\title{
Emerging X-by-Wire capabilities for military crew and platform protection
}

\section{David Abdulmasih*, John Melentis, Periklis Charchalakis and Elias Stipidis}

\author{
Vetronics Research Centre (VRC), \\ University of Brighton, \\ Brighton, BN2 4GJ, UK \\ Email: d.abdulmasih@vetronics.org \\ Email: e.stipidis@vetronics.org \\ *Corresponding author
}

\section{Simon Walters}

School of Computing, Engineering and Mathematics,

University of Brighton,

Brighton, BN2 4GJ, UK

Email: s.d.walters@brighton.ac.uk

\begin{abstract}
Improvised Explosive Devices (IEDs) have claimed many lives during recent wars. The response from military vehicle designers has been to provide more and more armour. However, this may not be the best long-term solution, as, amongst other factors, the weight of the vehicle increases dramatically. Aligning to the trend of more electric vehicles, this paper presents a different approach to tackle this problem which provides better protection for the crew, by removing the driver from the traditional physical location in the vehicle. A solution, such as Steer-by-Wire, leading to Drive-by-Wire crew station, can physically shift the location of the crew in the vehicle, thereby providing better protection against IEDs. This paper describes a procedure for designing such a system; it also describes a representative vehicle platform that has been assembled to demonstrate the proposed system functionality.
\end{abstract}

Keywords: DbW; Drive-by-Wire; active stick; decoupled crew station; survivability; crew protection; reliability; TTP; time triggered protocol; mobile demonstrator test bed.

Reference to this paper should be made as follows: Abdulmasih, D., Melentis, J., Charchalakis, P., Stipidis, E. and Walters, S. (XXXX) 'Emerging X-by-Wire capabilities for military crew and platform protection', Int. J. Vehicle Information and Communication System, Vol. X, No. Y, pp.xxx-xxx.

Biographical notes: David Abdulmasih is a Research Fellow at the Vetronics Research Centre at the University of Brighton, UK. He received his MEng (1st Class with Honours) in Computer Systems Engineering from the University of Sussex in 2009 and a PhD from University of Brighton in 2014. His research interests include modular vetronics architectures, vehicle networks and assurance of the integrity and reliability of the vehicle's electronic architecture.

John Melentis obtained MEng in Computer Systems Engineering and MSc in Satellite Communications \& Space Systems from University of Sussex in 2004 


\section{Abdulmasih et al.}

and 2006, respectively. He went on to obtain his $\mathrm{PhD}$ from University of Sussex in 2011. He joined industry thereafter working in the field of automotive electronics.

Periklis Charchalakis is a Principal Research Fellow and a Technical Manager at the Vetronics Research Centre at the University of Brighton, UK. He obtained a BEng in Electronics Engineering in 2000 and a $\mathrm{PhD}$ in 2005, both degrees from the University of Sussex. His research interests include embedded architectures, networks, military vehicle electronic architectures; and real-time and distributed systems application development.

Elias Stipidis is the Director of the Vetronics Research Centre and Professor of Vetronics at the University of Brighton, UK. He obtained a BEng (1st Class Honours) in Electronics in 1995 and $\mathrm{PhD}$ in 1998 from the University of Sussex. He is a Chartered Engineer, Fellow of the IET and a member of the VSI Steering Committee, GVA TWG, MilCAN TWG, and MILVA. His research interests include military integrated vetronics architectures, safety critical systems, automotive and X-by-Wire systems, verification and validation, distributed real-time systems, wireless ad-hoc networking and cluster formation, mobile networks, reconfigurable and intelligent control systems, real-time distributed software, and fault-tolerant techniques.

Simon Walters is a Principal Lecturer within the School of Computing, Engineering and Mathematics at the University of Brighton. He obtained an HND in Electronics Systems Engineering from Kingston Polytechnic in 1988. After two years in industry, he returned to university - at University of Brighton - and gained: a BEng (Honours) and an MEng in 1993, a PhD in 1998, and an MBA in 2003. His work is cross-disciplinary, spanning electrical, electronic, automotive, computer and mechanical engineering fields.

\section{Introduction}

New and irregular threats are imposed by the current operating conditions for military vehicles; these usually have severe effects on crew and platform survivability. Traditional methods focus on adding more armour and/or modifying the hull shape; however, this typically hinders the capabilities provided by the platform. A better approach is to allow the crew to be flexibly placed in a position with enhanced protection. Such approach can be achieved with an electronics-based control (X-by-Wire) capability. This paper focuses on the development and application of multi-crew station Steer-by-Wire (SbW) capability that will enable the enhanced protection of the crew and platform.

The remainder of this paper is organised as follows. Section 2 provides an overview of the related information highlighting the problem and current solutions and includes a brief review of related work. Section 3 introduces the multi-crew station SbW capability and includes requirements representing the need for this capability and a proposed design based on those requirements. The implementation of a case-study demonstrator is described in Section 4 and the testing and results of the demonstrator are further discussed. Finally, some concluding remarks are provided in Section 5. 


\section{Emerging X-by-Wire capabilities}

The work, described in this paper, started as a collaborative effort between the Authors in the Vetronics Research Centre (VRC) and Ricardo UK to de-risk Steer-byWire for the UK Future Protected Vehicle program, and has been continuously evolving to a mature and modular solution for enhanced platform and crew survivability.

\section{The military need for Drive-by-Wire (DbW)}

The military sector views $\mathrm{DbW}$ as an emerging technology with many benefits to offer (Hodgson and Make-Kail, 2009). The following sections elaborate on multiple scenarios where $\mathrm{DbW}$ is very important. As western armies have to face a continuously expanding asymmetric threat, land forces cannot confront the opposing force (OPFOR) in a traditional manner. Between 2006 and 2011, Improvised Explosive Devices (IEDs) and other Improvised Munitions (IM) were responsible for 1141 fatalities in Coalition Forces in Iraq and another 1166 in Afghanistan, which contributes to more than $60 \%$ of all hostile fatalities in Iraq and about 54\% in Afghanistan (iCasualties.org, 2016). Therefore, new innovative technologies are required to help the fighting personnel remain safe against guerrilla warfare in an urban warzone setting.

In a typical IED attack, the IEDs are planted along a route that allied patrol vehicles use. The IED usually explodes into the front underside of the vehicle, directing the full force of the blast at the front wheels and the driver.

In the event of such a blast, the steering column and steering wheel assembly is often dislocated from its mounting and travels towards the driver, often with lethal effect, as shown in Figure 1 (Ruhlen, 2009). Note that the vehicle in the figure is actually an MRAP (a purpose-built, Mine-Resistant Ambush Protected truck). This means that the vehicle was specifically designed to counter such threats - and in this case, the crew survived.

Figure 1 The effect of an IED blast (note the steering wheel)

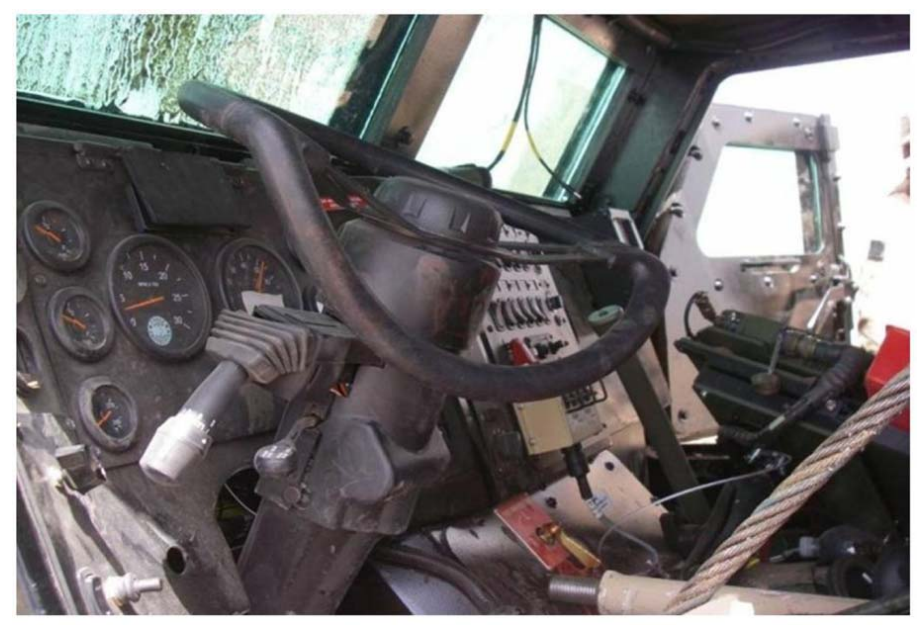

As mentioned before, one solution to this problem is to keep adding more and more armour and designing a V-shape into the vehicle, which indeed provides some positive 


\section{Abdulmasih et al.}

results, as the figure shows. However, the problem with this approach is twofold. (1) Whenever the OPFOR realises that their IEDs are relatively ineffective, they just double the explosive mass; a quite-effective 'low-tech' solution. (2) The extra armour is, by definition, a very heavy solution, which means an underpowered vehicle, which wasn't designed to handle the extra weight. This means that the vehicle lacks the ability to accelerate quickly out of a problematic situation. The V-shape structure, used underneath the vehicle as a countermeasure to deflect the blast sideways, raises the vehicle's centre of gravity, thereby increasing the risk of the vehicle overturning during violent manoeuvring (Clay, 2011; Mitchell and Scutro, 2011). With the added weight, bridge crossing, driving through soft/weak roads and mud crossing also become substantial issues. These factors equate to greatly reduce effective range and capability of the vehicle, with additional disadvantages of higher fuel consumption and costs.

When used as a technology enabler for crew survivability, Drive-by-Wire (DbW) physically separates the operator from the actuators of the vehicle; it enables a different physical position for the driver, which can be better protected; the lack of protection is a cause of many casualties and fatalities in the patrol vehicles. This can ultimately provide the steppingstone to a crew station that is internally suspended in the vehicle, designed to absorb the rapidly expanding gases and concussive effect of the exploding IEDs, IMs, or other land mines.

Implementing a $\mathrm{DbW}$ solution provides several additional benefits, which arise from a systems engineering perspective.

In the scope of military vehicles, such as a truck, a patrol vehicle, or Armoured Personnel Carrier (APC), the driver is required to be at the very front of the vehicle, due to the mechanically coupled controls; the most obvious example is steering and the steering column and pedals. This poses several problems for the system designer, as the requirement to keep the driver in the front of the vehicle imposes several constraints, to which the designer must find a solution, usually by duplicating equipment to cover the following requirements:

a) On-board blast/ballistic crew protection;

b) Communication, into, and out of the vehicle;

c) Extended electronic clusters, with duplicate screens for the driver and the rest of the crew;

d) Extended life-support measures, such as Heating Ventilation Air Conditioning (HVAC);

e) Visibility, especially for Armoured Fighting Vehicles.

$\mathrm{DbW}$ can tackle these issues, and provide a defence against enemy action; if the enemy aims to immobilise an armoured vehicle by attacking the driver, they would not know the exact location of the driver. In addition, $\mathrm{DbW}$ can counter crew fatigue when driving in rough terrain by minimising the effect the outside environment has on the steered wheels, and consequently the feedback the driver receives from the handwheel.

$\mathrm{DbW}$, in the military context, provides a means for increasing crew protection, while concurrently expanding on the on-board capabilities. Using additional control inputs, such as joysticks, a $\mathrm{DbW} \&$ stick combination can be used, to demonstrate mission versatility; solutions such as remote control of a vehicle would also be feasible. Remote control is the ability of the crew, based in a secure bunker or in a different vehicle, to 


\section{Emerging X-by-Wire capabilities}

operate another vehicle remotely. Tele-operated vehicles are gaining momentum and popularity, as they can enter a high-risk area without risk to human life. A DbW \& stick in such a vehicle would enable a rapid changeover from a local human operator to a remote one.

There are several efforts that aim to exploit the benefits of $\mathrm{DbW}$ in military vehicles. These efforts span the full spectrum of development from early research work to actual military products. One piece of research related to this work was carried out by Mushenski et al. (2003), which looked at the top-level design of an automotive DbW architecture for military ground-based vehicles. The work included a digital data-bus, based on Controller Area Network (CAN) protocol, with an analogue wired backup system for vehicle control of the throttle, braking and steering functions. The work also provided early discussions of the failure modes of the key functional elements of the DbW system. On the other end of the spectrum, the BAE Terrier Combat Engineering (tracked) Vehicle is considered the first combat vehicle to feature Drive-by-Wire technology (BAE Systems, 2016). The Terrier can be fully controlled via the commander's joystick through the DbW systems and the front loader and excavator arm are controlled through a digital data-bus. The Terrier can also be remotely controlled using gaming type controller and remote cameras. However, for safety reasons, physical connections are maintained in the Terrier between the driver and the automotive/ powertrain system.

\section{Multi-crew station SbW capability for military vehicle}

\subsection{Requirements analysis}

The functional requirements for a $\mathrm{DbW}$ system are divided into top-level and subsystem specific requirements. The top-level requirements are aimed at meeting open standards and recommendations of the Generic Vehicle Architecture (GVA) (UK MoD, 2010). This includes taking into account a modular and generic approach, which is flexible and scalable, allowing for through-life capability management (TLCM). Conversely, the subsystem specific requirements are focused on the generic SbW capability in a generic automotive environment, as a prime subsystem of $\mathrm{DbW}$. In military terms, this generic approach allows the capability and resource selection for a cross-platform deployment on both wheeled and tracked vehicles with either conventional or hybrid drivetrains. Examples of the GVA-related DbW requirements are:

- Multi-crew station integration with applicability for different vehicles;

- Vehicle operational and environmental configuration;

- Logical subsystem distribution over integrated vetronics;

- Multi-crew station integration allowing more than one input on the same vehicle;

- Mobility, i.e. DbW subsystem integration with tactical manoeuvre capability;

- Semi-autonomous functions with various feedback options;

- Certification (e.g. reusability of components for safety cases and different legislation);

- Other subsystem integration (e.g. Health \& Usage Monitoring Systems). 


\section{Abdulmasih et al.}

Specific functional SbW requirements (note: many of these assume two steering inputs, e.g. driver and commander):

- Should provide mechanical means of yawing the vehicle via steering controls, hereafter mentioned as steered wheels;

- Should be able to conduct steering swap capability whether the vehicle is in motion or stationary;

- Crew controlling the vehicle are named as driver and commander;

- The commander shall assume the driving role, if the driver cannot perform the task;

- The commander may have no visual reference to the driver's controls;

- Primary driver controller must be a rotational position sensor, named hereafter as a steering wheel;

- Secondary controller should be a linear position or force sensor, named hereafter as a joystick;

- Steering wheel and joystick positions should be absolute, in relation to the steered wheels.

\subsection{Design of multi-crew station $\mathrm{SbW}$}

The role of an Armoured Fighting Vehicle (AFV) has evolved dramatically over the last few decades. It is envisaged that near-future missions will focus on the need for an agile platform that is rapidly deployable, configurable, highly survivable, and costeffective. Weight and mobility are counteracting factors, particularly when it comes to survivability. Nevertheless, these types of trade-offs offer the challenges that a fully integrated vehicle Electronic Architecture (EA) could mitigate against and, furthermore, provide a through-life capable, highly mobile, flexible, highly survivable, mission adaptable and, when needed, highly lethal platform.

Defence Standard 23-09 (GVA), drawn from research on Vehicle Systems Integration (VSI) standards and guidelines (Connor, 2009), offers an informative guide on a Future Protected Vehicle (FPV) Electronics Architecture (EA) design. This research considers future platforms, introducing some new concepts and considerations. We recommend that the main assumptions for the Generic FPV EA to be:

a) Modular platform with multiple capabilities through standard interfaces;

b) Engine/chassis that can potentially support vehicle separation into manned and unmanned sections, with drivetrain for wheeled or tracked mobility, articulation, potential hybrid, etc.;

c) Integrated survivability;

d) Standardised cross-fleet main platform capabilities (e.g. Government Furnished Equipment);

e) Mission modularity.

A generic, modular solution is envisaged in the form of layers to cover the aforementioned requirements. 


\section{Emerging X-by-Wire capabilities}

Each of the layers, shown in Table 1, has its own defined characteristics. The SbW Subsystem higher layers (Application and System) provide service-oriented commonality for different platforms, while the lower layers (Class and Vehicle) define figurative and more specific functionality. The Abstraction layer is a middleware set of functions to interconnect the generic SbW architecture with the specific vehicle class type and physical subsystem.

Table $1 \quad$ SbW generic architecture layered approach

\begin{tabular}{|c|c|}
\hline Layer & Function \\
\hline Application layer & $\begin{array}{l}\text { Induce a human-controlled yaw moment in the vehicle, user defined vehicle } \\
\text { operation for civil and defence environment (includes automotive and military } \\
\text { associated functionality) }\end{array}$ \\
\hline System layer & $\begin{array}{l}\text { SbW (including Torque Vectoring) Control Algorithms; this would be further } \\
\text { expanded to include other XbW (e.g. brake and throttle) and military modules } \\
\text { (e.g. related to survivability, lethality, etc.) }\end{array}$ \\
\hline Abstraction layer & $\begin{array}{l}\text { Standard Interfaces (mapping functionalities between the system and class } \\
\text { layers) }\end{array}$ \\
\hline \multirow[t]{13}{*}{ Class layer } & Wheeled or Tracked \\
\hline & Steering Actuation \\
\hline & Enemy Action Resilience \\
\hline & Occupant Control Takeover \& deconfliction \\
\hline & Roadworthiness Monitoring \\
\hline & Mission Readiness Monitoring \\
\hline & Survivability Envelopes \\
\hline & Faulty Actuation Mitigation \\
\hline & Health \& Usage Monitoring Systems (HUMS) \\
\hline & Electronic/Maintenance checklists \\
\hline & (Semi)autonomous drive depending on requirements \\
\hline & Test Drive (repeatable, in circuit) \\
\hline & Hybrid Electric Drive (HED) \\
\hline \multirow[t]{6}{*}{ Vehicle layer } & HMI Set-Point Generators - Joystick/Handwheel \\
\hline & Control Gains \\
\hline & System Responses \\
\hline & Torque Settings \\
\hline & Monitoring \\
\hline & Driver profiles \\
\hline
\end{tabular}

Focusing on Figure 2, the Application layer in this instance is SbW and hence by definition its own characteristic is to steer the vehicle. Further detailed characteristics can be added according to the requirements on the SbW Application Layer which, for example, could be the capability to steer the vehicle from more than one crew station. To accomplish this, the System layer is used to utilise inputs (set-point generators with force feedback) alongside further automotive inputs (feedback control) and outputs (actuators) available at the vehicle (services), all interconnected through a common EA (an integrated network) to produce the by-wire system. 
D. Abdulmasih et al.

Figure 2 SbW subsytem layer design

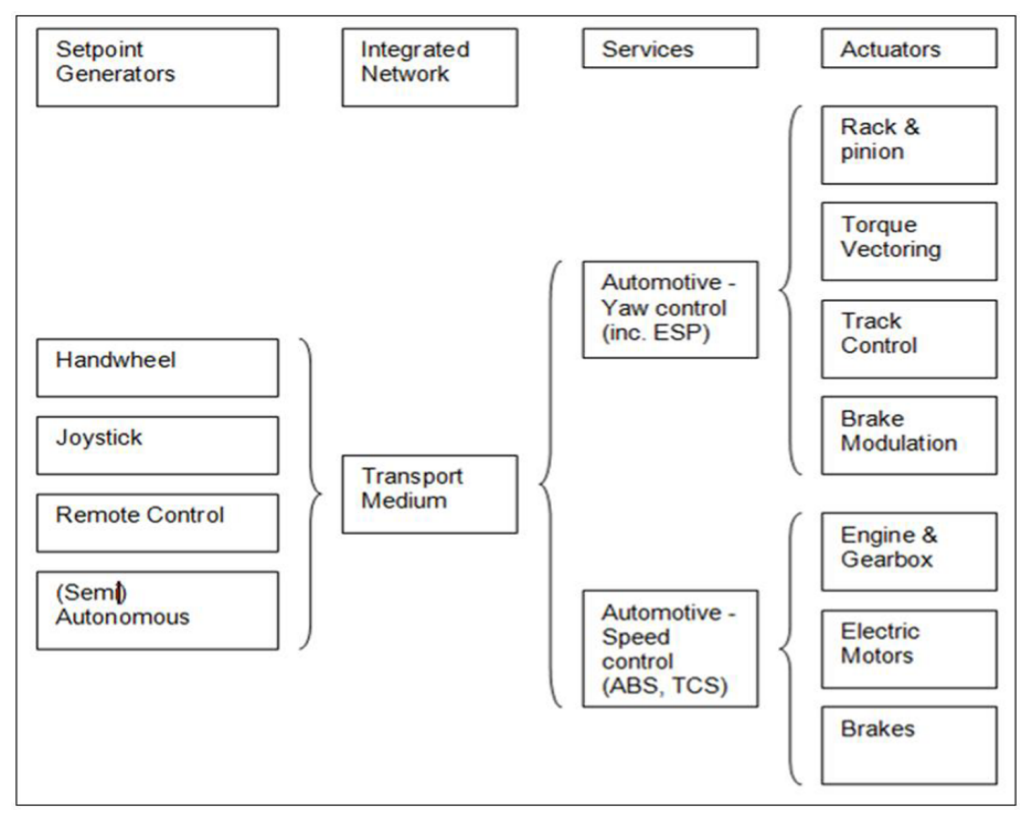

Figure 3 SbW physical subsystem

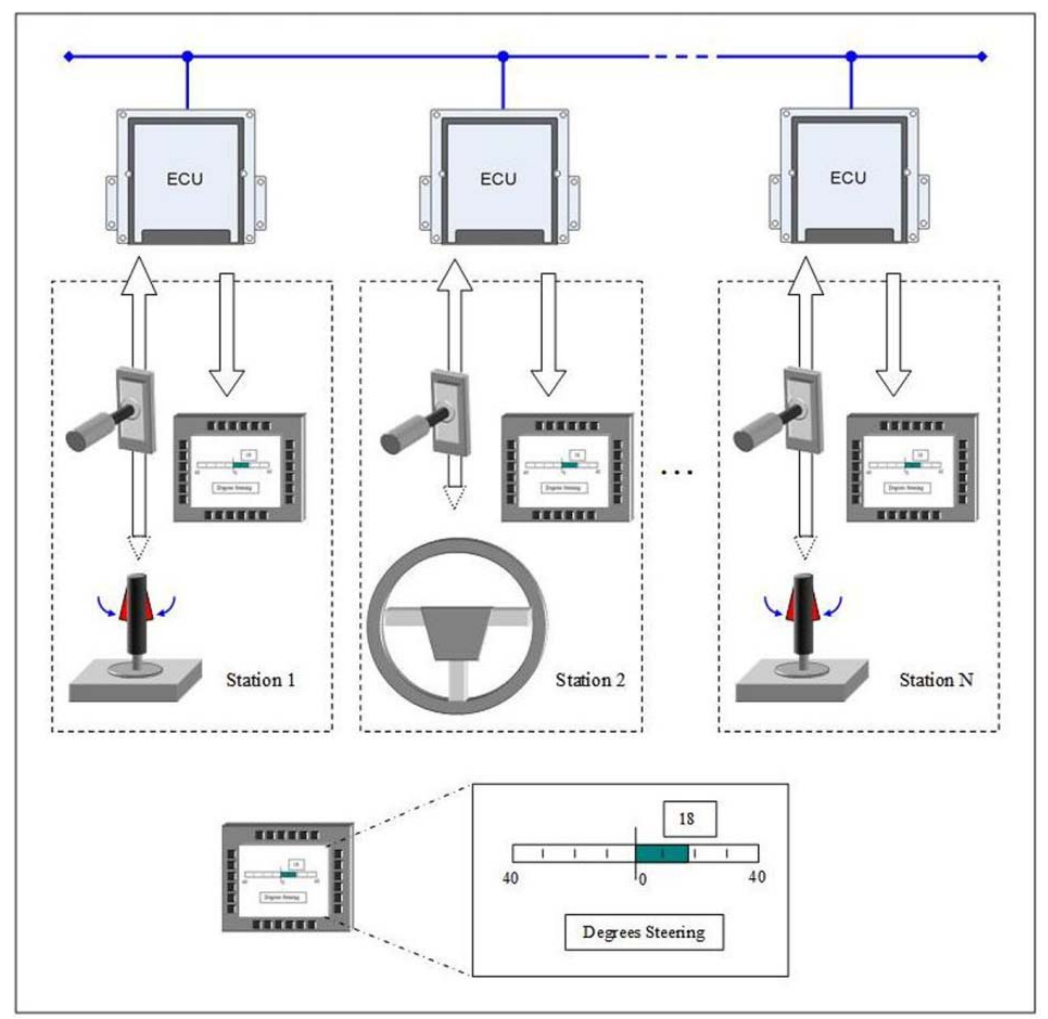




\section{Emerging X-by-Wire capabilities}

As mentioned previously, the logical subsystem design is technology independent and unrelated to the physical layout of the EA. To design, test and verify/validate the SbW Physical Subsystem, it is necessary to define the actual vehicle and technology insertion, including the EA topology. The assumptions are that whatever the physical layout, the SbW subsystem should use a suitably reliable technology, which would satisfy the safety requirements of the $\mathrm{SbW}$ application, to interface with (and perhaps to partition $\mathrm{SbW}$ from) the rest of the vehicle's electronic architecture. As a top-level example, the physical subsystem design for $\mathrm{SbW}$ is depicted in Figure 3.

Figure 3 illustrates how the requirements given in Section 2 can be met. A number of stations capable of SbW can be introduced to the vehicle. All can be interfaced to the safety critical network via Electronic Control Units (ECUs) transparently and with different mechanical inputs, e.g. Steering Wheels or Joysticks. Mechanical force feedback is also assumed for the design, as well as visual feedback in terms of the absolute relation of the steering input and steered wheels. Equivalent technology ECUs are assumed to exist towards the end system for the steered wheel actuators.

The following stages were identified and implemented for testing and verifying/ validating the design, with Risk Management introduced into the process and system certification (Philippi, 2002):

1 Modelling and simulation of the SbW in a suitable environment;

2 Applying fault analysis (e.g. Failure Modes \& Effect Analysis [FMEA]) (US DoD, 1980) to the model using the Ricardo AutoFMEA tool (Ricardo AutoFMEA, 2011);

3 Interfacing the model to safety-critical technology (hardware in the loop);

4 Porting SbW software onto hardware that can be certified up to the required Safety Integrity Level (SIL).

To verify and validate the Generic SbW Architecture as a logical system, a case study was selected (VRC, 2012; Summers et al., 2009), the 'Buggy' VSI mobile demonstrator platform, detailed in the next section.

\section{Case study: the mobile demonstrator test bed}

It is important to note that this work only considers safety analysis through FMEA and does not cover the safety cases for this system. As per X-by-Wire Project (1998), in order to effectively verify and validate a safety-critical $\mathrm{XbW}$ system for certification and legislation purposes, fault injection should be employed, perhaps to support arguments presented within the safety cases.

This study took advantage of previous work performed by the VRC mobile vetronics validation demonstrator 'Buggy'. The Buggy, shown in Figure 4, is a Commercial Offthe-Shelf (COTS) wheeled two-person, 600cc Internal Combustion (IC) Engine, off-road mobile demonstrator; amongst its features are conventional automotive controls that have been modified for $\mathrm{DbW}$ and electronic connections modified and integrated to other $\mathrm{XbW}$ subsystems. This demonstrator was designed to be a validation platform for Vetronics Integration (only on a network level) following the VSI standards and guidelines (Connor, 2009). Applications include DbW, Local Situation Awareness (LSA), HUMS, Automotive Utilities, Crew Station and Vehicle Management, and Telemetry 


\section{Abdulmasih et al.}

with remote wireless Real-Time Video Streaming. Technologies inserted to support these applications include: Time-Triggered Protocol (TTP) (Pimentel and Sacristan, 2001), MilCAN (Summers et al., 2006), Ethernet, and WLAN (Nolte et al., 2005).

An additional controller input, representing a second crew station, has been implemented for SbW in the Buggy. This covers the architectural framework, of the type of equipment and software algorithms that could be used to design such a subsystem and covers potential switchover policies between SbW controllers. The architectural framework also includes the proposed implementation of how the system designer could apply this to a military vehicle and an actual proof-of-concept solution for the Buggy.

\subsection{Electronic architecture}

The electronic, service-oriented architecture for the Buggy is shown in Figure 5. It is a proof-of-concept design extension of the test bed presented in Summers et al. (2009). It is clear that the design of the buggy architecture is based on the top-level design requirements mentioned in Section 3, but tailored to meet the needs of this research. The existing mechanical, electrical, and electronic connections were modified for $\mathrm{DbW}$ capability, using TTP and Health Monitoring \& Management (HMM) (Melentis et al., 2008), and a utility segment for MilCAN, towards a Service-Oriented Architecture (SOA). All subsystems are fully integrated through VSI Bridges (Charchalakis et al., 2006) acting as gateways enabled through middleware and standard interfaces to the rest of the platform, to the extent that when the brakes are applied (TTP), the brake lights on the back of the vehicle switch on (MilCAN), and all this information is available (VSI Bridge) wirelessly (WiFi access point) as telemetry and HUMS data.

Figure 4 The electronic architecture of the buggy (see online version for colours)

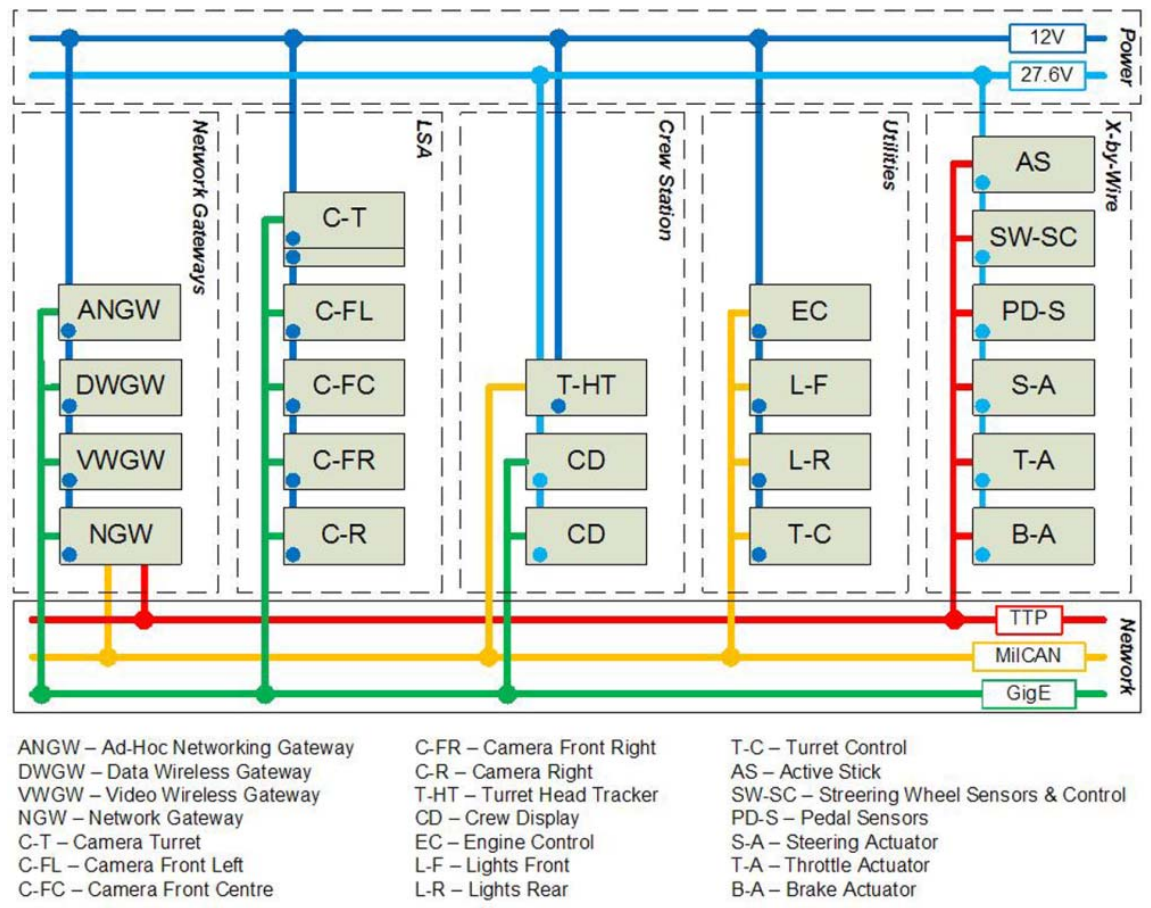




\section{Emerging X-by-Wire capabilities}

In this case study, the user requirement for supporting multiple crew station inputs has been translated accordingly into the system design through appropriate modelling and simulation as per the generic architecture design approach. The model was then implemented and interfaced with the TTP as an example of technology insertion for the DbW application.

The DbW system consists of three subsystems: the Throttle-by-Wire (TbW), the Brake-by-Wire $(\mathrm{BbW})$, and the Steer-by-Wire (SbW). TTP nodes handle all communications regarding $\mathrm{DbW}$, as shown in Figure 5.

Figure 5 The DbW architecture of the buggy
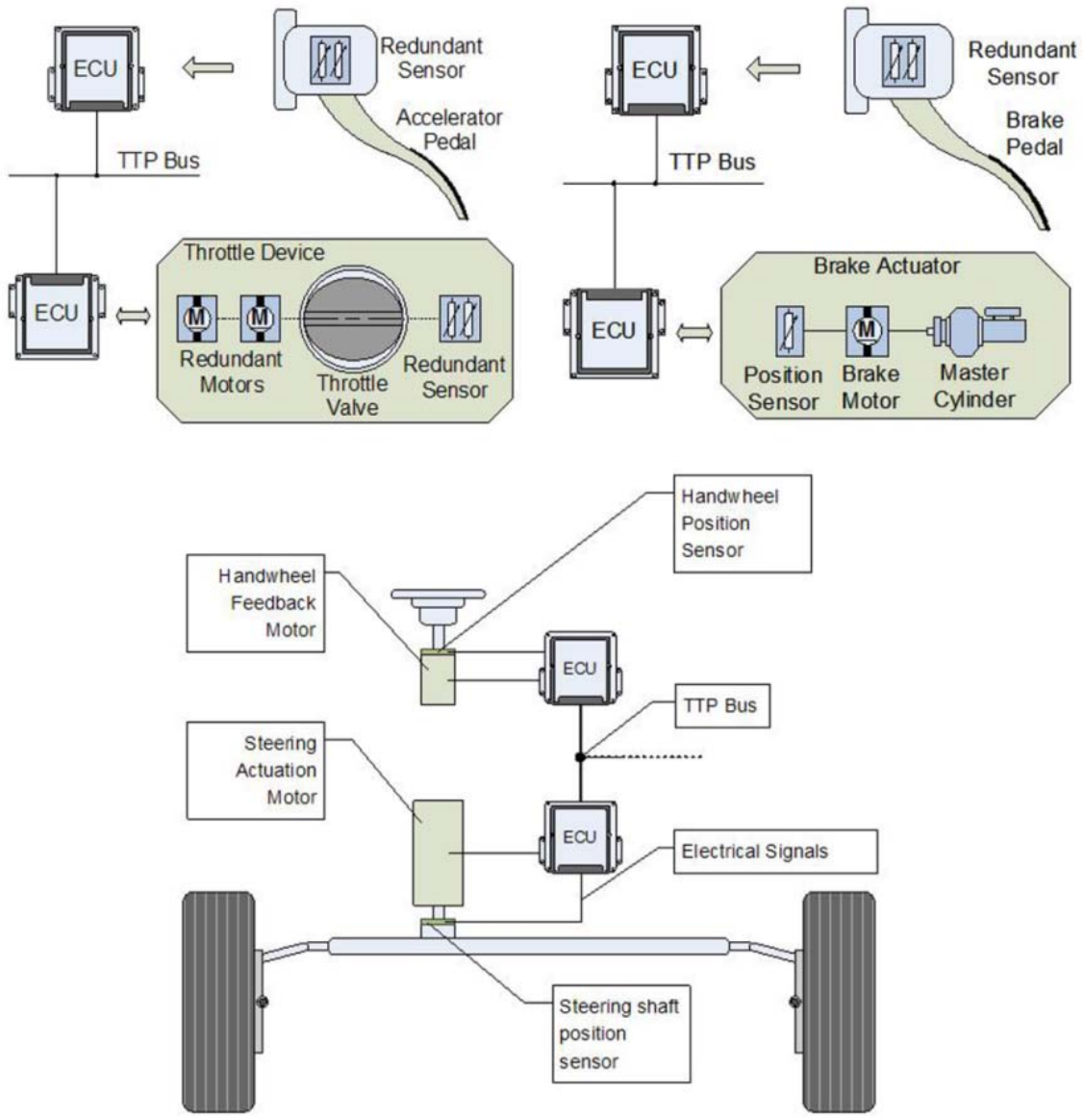

Figure 6 illustrates the generic physical SbW subsystem where crew stations for 'Driver' and 'Commander' were adopted on the Buggy and integrated with an existing TTP network node. The driver's input includes monitoring of the SbW system activity through a dedicated display, whereas the Commander's steer input can take over the steering, according to a $\mathrm{SbW}$ arbitration algorithm. 
D. Abdulmasih et al.

Figure 6 The active stick subsystem

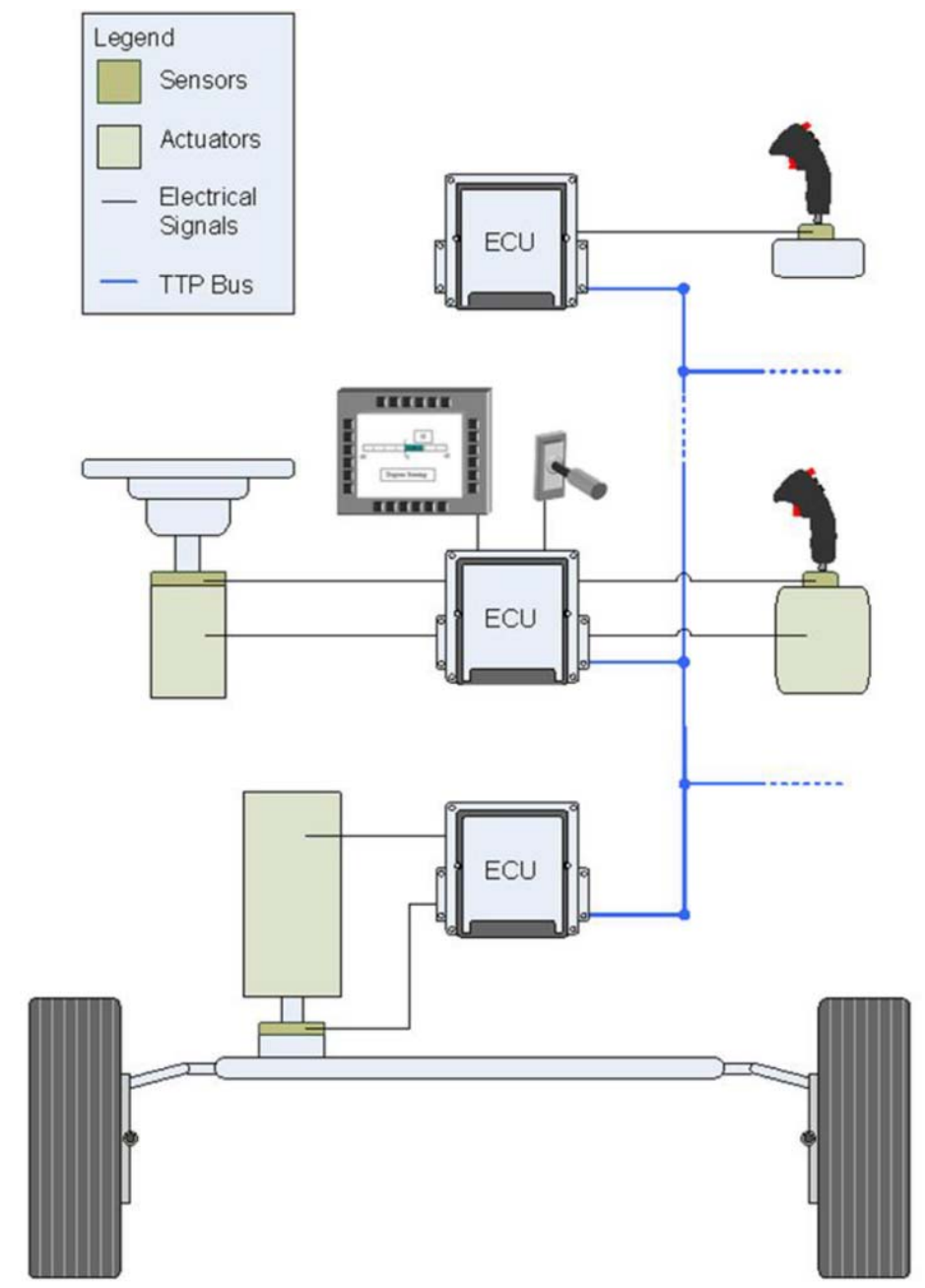

\subsection{Failure modes \& effect analysis (FMEA)}

An FMEA analysis has been carried out using the Ricardo AutoFMEA tool. The AutoFMEA tool is part of a MATLAB Simulink-based approach to safety analysis. The analysis aims to identify the main hazards associated with the generic architecture. Since this paper is only concerned with the Steer-by-Wire capability, a top-level design of a SbW system is modelled in Simulink; this model is based on the SbW logical subsystem design discussed earlier in the section on logical subsystem design. Various AutoFMEA elements are added in order to carry out the analysis. The top view of this model is shown in Figure 7 and the various subsystems are discussed further. 


\section{Emerging X-by-Wire capabilities}

Figure 7 AutoFMEA - the top-level SbW model

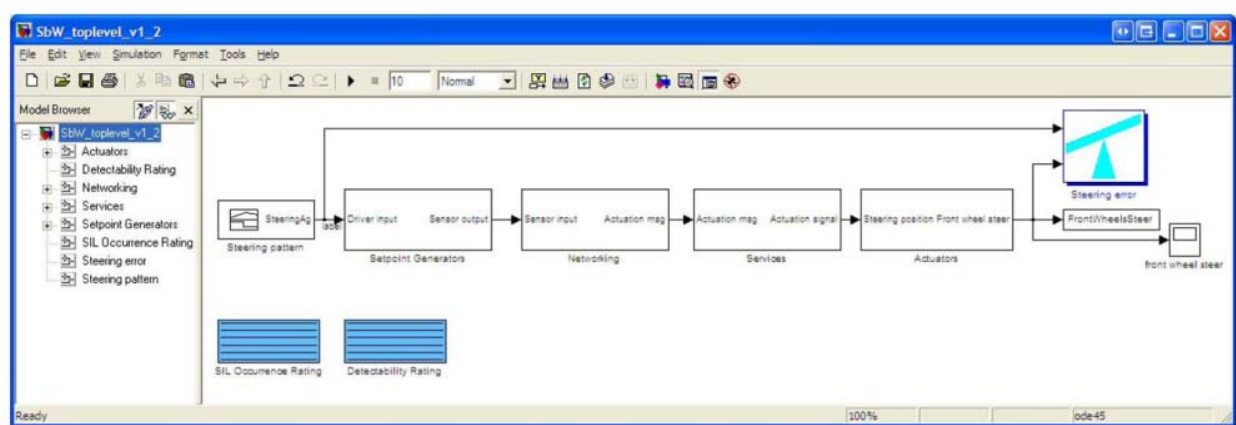

An occurrence ratings table was used to specify the probability intervals of effect occurrence. Likewise, a detectability ratings table was used to specify the probability intervals of effect detectability. These tables provide occurrence and detectability rankings (each between 1 and 10) for each effect. Meanwhile, for the steering error effect a lookup table was specified so that the severity of the steering error is a function of the difference between the requested steering position and the achieved steering positions. This function can be described as:

$$
\text { Severity }=(\mid \text { Requested position }- \text { Achieved position } \mid) / 2
$$

and rounded up to the nearest integer. The Steering pattern block simulates a typical steering input. The set-point generators subsystem contains the steering sensor and the different failure modes as presented in Figure 8.

Figure 8 The set-point generators subsystem

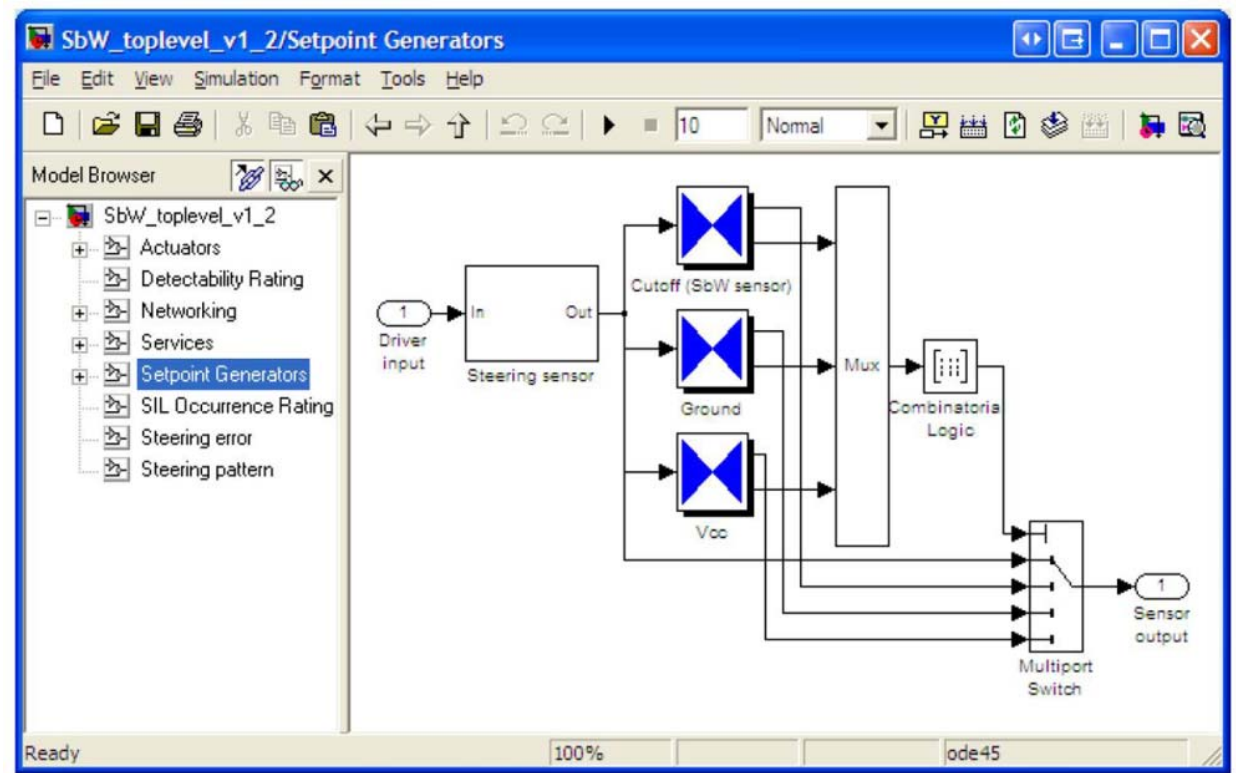




\section{Abdulmasih et al.}

Three failure modes are simulated, these are:

- Cut-off: the steering sensor is in an open-circuit state and hence it returns the last valid value;

- Ground: the sensor is short circuited to ground;

- Vcc: the sensor is short circuited to Vec (5V).

A typical failure rate of 7.91e-10/sec (Barbier and Gilhead, 2005) was assumed for the sensor and to simulate worst case scenario this value is used for each failure.

The Networking subsystem simulates a typical network topology, shown in Figure 9, where the steering sensor reading is fed into an ECU which communicates with another ECU (actuator side) over a network bus.

Figure 9 The networking component of the model

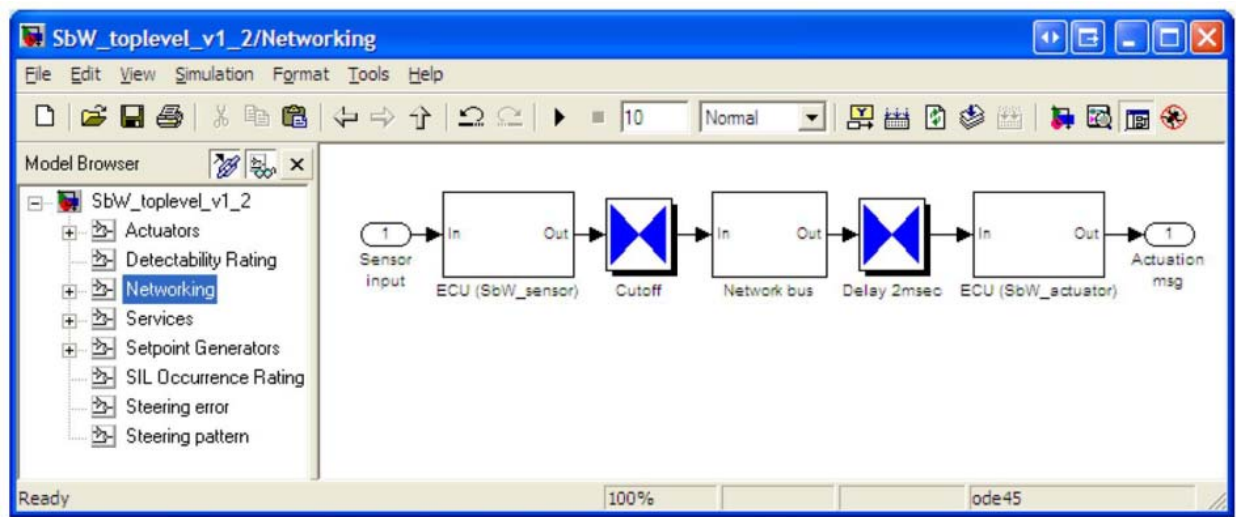

Two failure modes are added, these are:

1 Cut-off: represents failure of the ECU. The failure rate of the TTP ECU is used as a typical value, it has a failure rate between $10 \mathrm{e}-7 / \mathrm{sec}$ and $10 \mathrm{e}-6 / \mathrm{sec}$ (TTTech Computertechnik, 2007); hence, the mean value of $5.5 \mathrm{e}-6 / \mathrm{sec}$ is used.

2 Delay: this simulates the delay caused by the network. An average typical value for TTP network is $2 \mathrm{msec}$ (Summers et al., 2009); however, this is present all the time and hence it has failure rate of 1 .

The Actuators subsystem in Figure 10 contains the steering actuator/motor and couple of perceived failures.

The failure modes are:

1 Multiplicative Noise: this simulates signal interference experienced by the actuators that might alter their operation. A typical signal variation of 0.4 at a rate of $2 \mathrm{e}-14 / \mathrm{sec}$ is assumed.

2 Cut-off: this simulates the loss of actuator action with a failure rate of $5.56 \mathrm{e}-13 / \mathrm{sec}$. 
Figure 10 The actuators subsystem

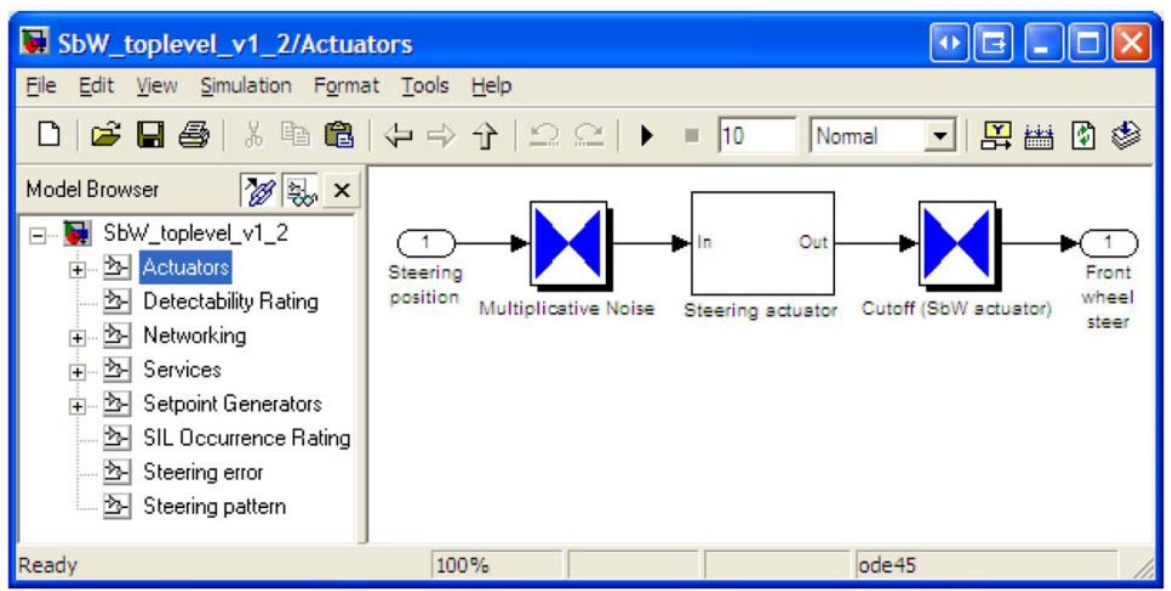

\subsection{Evaluation}

The test plan for the buggy included the following:

- Testing the input for steering (handwheel and controller) and the arbitration algorithm;

- Results from the TTP bus, captured in real time;

- Data analysis for the SbW subsystem, including accuracy and responsiveness;

- Feedback from test drives and demonstrations.

The testing methodology is based on the TTP bus monitoring software, TTP-View (TTTech Computertechnik, 2011). Information from the TTP bus is shown to the PC via the TTP Monitoring Node (a TTP to Ethernet interface) using an Ethernet connection. The monitoring data are recorded and saved as a file, which the developer can then playback in a similar fashion to a video tape recording.

In Figure 11, the interaction between the joystick and handwheel is shown. The vertical axis in the top graph shows the position of the set-point generators, as a percentage. The vertical axis in the bottom graph represents the state of the override switch and override buttons. The horizontal axis for both graphs describes time in TDMA cycles, with the leftmost reading being the oldest (20,000 TDMA cycles * $(2400 \mu \mathrm{s} / \mathrm{TDMA}$ cycle $)=48$ seconds $)$ and the right-hand reading is the most recent. There are several time points of note, shown in the graphs as points A-E, which are described in detail later on. The horizontal grid is also set to 1000 TDMA cycles.

In the top graph, the vertical axis scale is a percentage $0 \%$ means full left, $50 \%$ is centre, and $100 \%$ stands for full right. Three values are shown:

1 Steer_joy_witt_pos is the current position of the joystick;

2 Steer_shaft_pos is the current position of the handwheel;

3 Steer_wheels_pos is the current position of the wheels. 


\section{Abdulmasih et al.}

It is evident that the closer the handwheel position is to that of the wheels, the smaller the error, and the faster the system response.

Figure 11 Multiple controller results
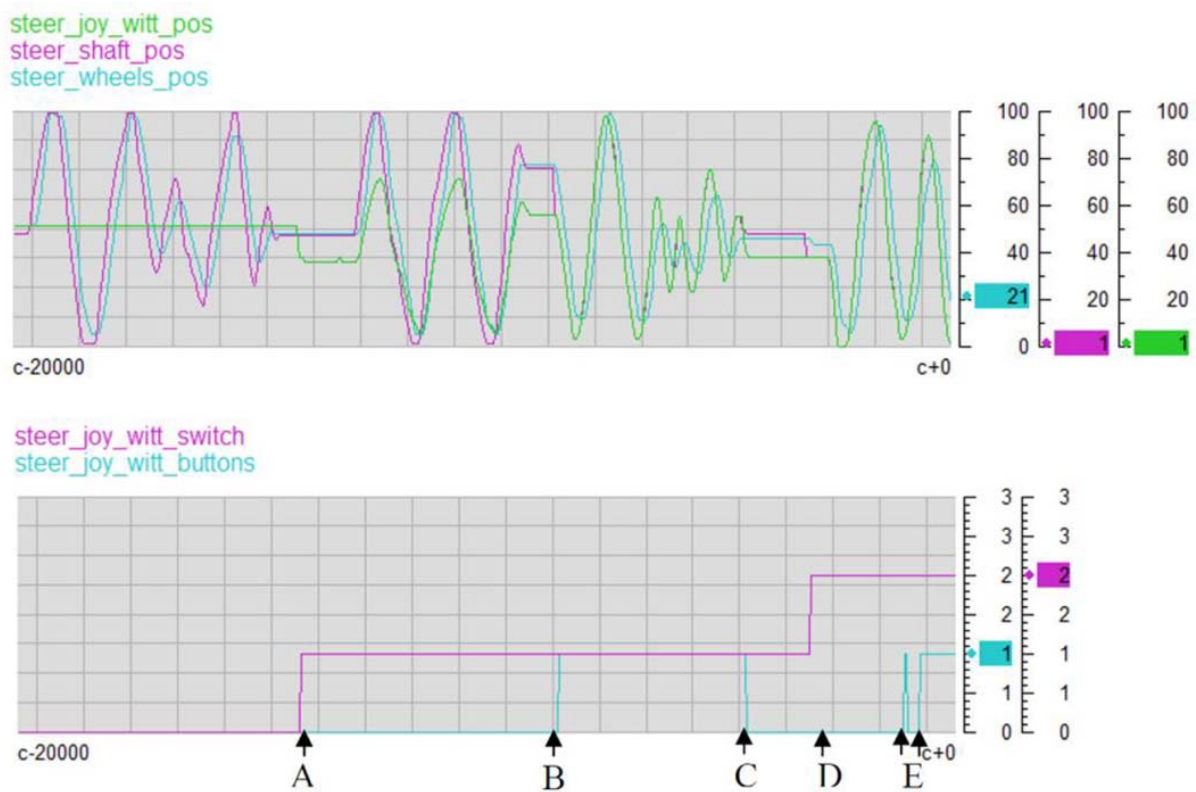

In the bottom graph, the current status of the switches and buttons is shown, as numbers, to differentiate between the different states. Two values are measured here:

1 Steer_joy_witt_switch represents the current state of the switch: ' 0 ' for 'OFF' (the joystick is disabled), ' 1 ' for STBY or passive mode (the joystick follows the handwheel) and ' 2 ' for 'ACT' or active mode;

2 Steer_joy_witt_buttons represents the temporary override buttons on the joystick. ' 0 ' means no buttons are pressed, and ' 1 ' means that both buttons are pressed. Note that if only one of the buttons is pressed, the system will still show the result as ' 0 '.

In the time before ' $\mathrm{A}$ ', where the switch is off and no buttons are pressed, the wheels follow the handwheel, while the joystick does not move. At time point $A$, when the switch is set to 'STBY', the joystick moves to follow the handwheel. Note that there is a small error between the two, which is derived from the calibration routine of the position feedback in the joystick, and varies slightly every time the system is turned on.

At time point 'B', the temporary buttons in the joystick are activated, while the switch is still in STBY mode. This shows that the handwheel and the wheels immediately follow the position of the joystick. This continues until time point ' $\mathrm{C}$ ' where the buttons are released. After this point, the system behaves exactly as for after point 'A'.

At time point ' $\mathrm{D}$ ', the switch is changed to ACT mode, and handwheel and wheels follow the joystick, irrespective of what the buttons do. As an example, button press-andrelease cycles are introduced at time point ' $E$ ', showing no change in the system behaviour. 


\section{Emerging X-by-Wire capabilities}

The AutoFMEA analysis was carried out and it included all of the failures discussed earlier; however, a maximum of three was set as the number of failures to be present at any point of time. The solver was set to compute probabilities for a year (365 days).

The AutoFMEA analysis showed that the primary sources of concern arise from the ECU and steering sensor failures as they have the highest Risk Priority Number (RPN) value of 150. Moreover, the combination of actuator failure and network ECU failure contributed to an RPN value of 105, as shown in Table 2. Table 2 is a summary of the AutoFMEA results that shows the most significant failures (failures with the highest RPN).

Table 2 The AutoFMEA analysis results

\begin{tabular}{cllc}
\hline No. & Potential failure mode & Potential effect of failure & $R P N$ \\
\hline 1 & $\begin{array}{l}\text { Networking failure (cut-off and delay) + steering } \\
\text { sensor failure (cut-off or short circuit) }\end{array}$ & Steering error & 150 \\
\hline 2 & Networking failure (cut-off and delay) & Steering error & 105 \\
\hline 3 & $\begin{array}{l}\text { Steering actuator failure (cut-off) + networking } \\
\text { failure (cut-off and delay) }\end{array}$ & Steering error & 105 \\
\hline 4 & $\begin{array}{l}\text { Steering sensor failure (cut-off and short circuit) }+ \\
\text { networking failure (delay) }\end{array}$ & Steering error & 105 \\
\hline 5 & $\begin{array}{l}\text { Steering actuator failure (noise) + networking } \\
\text { failure (cut-off and delay) }\end{array}$ & Steering error & 45 \\
\hline 6 & Networking failure (delay) & Steering error & 45 \\
\hline 7 & $\begin{array}{l}\text { Steering actuator failure (cut-off and noise) }+ \\
\text { networking failure (cut-off or delay) }\end{array}$ & Steering error & 15 \\
\hline 8 & $\begin{array}{l}\text { Steering actuator failure (cut-off and noise) }+ \\
\text { steering sensor failure (cut-off or short circuit) }\end{array}$ & Steering error & 15 \\
\hline 9 & Steering actuator failure (cut-off and noise) & Steering error & 15 \\
\hline
\end{tabular}

\section{Conclusions}

The results show that the system and the interaction between the joystick and the handwheel perform as expected. Figure 12 shows the buggy during testing with the driver performing the steering by using the joystick.

One of the interesting findings of this work is the speed of development. Cumulatively, the multiple crew station SbW and the AutoFMEA analysis took less than three months to develop and implement. This time-frame included typical procurement issues where the feedback joystick arrived very late in the development. This is indicative of the benefits of an integrated and scalable design for the vehicle electronic architecture which enables extra capabilities to be rapidly deployed in a vehicle. Indeed, such a tactic is very useful as a quick and cost-effective response to Urgent Operational Requirements (UORs).

The modularity of this approach offers a flexible way to add further functionality integrated within the system such as Drive-by-Wire (DbW), whereby the Application layer would include the Brake and Throttle, with the DbW System layer encompassing (using peer-to-peer interconnection) the System layers of Steer, Brake, and Throttle. 


\section{Abdulmasih et al.}

Figure 12 The joystick used as steering input while driving

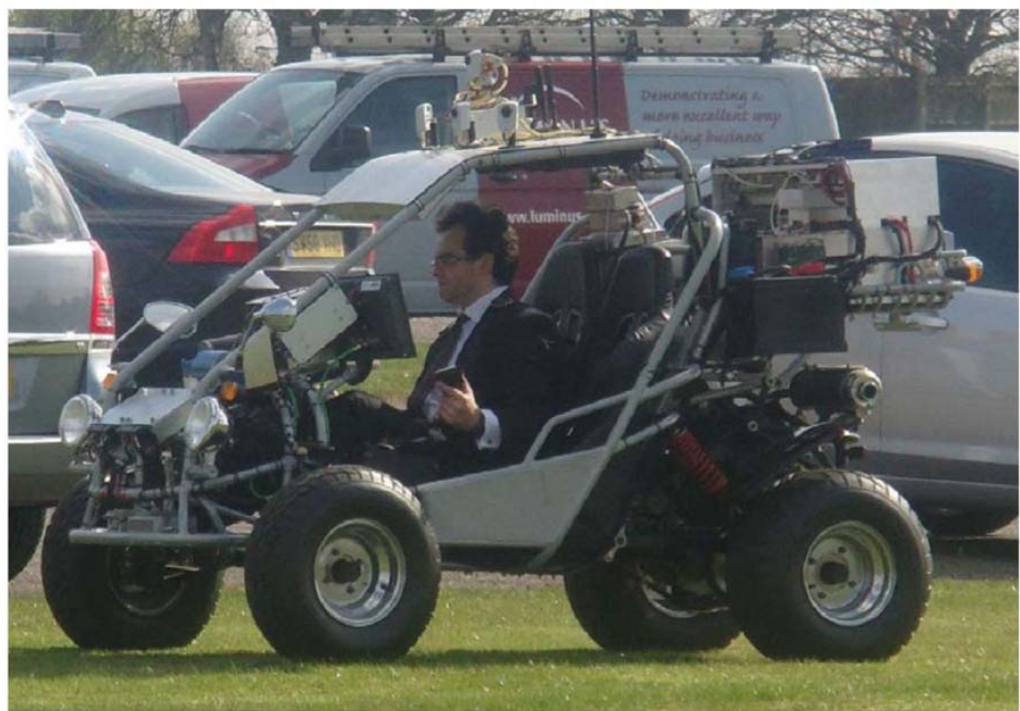

This work has highlighted the benefits that can be attained from a DbW in military vehicles. Essential to the realisation of a DbW system is a safe and reliable vehicle communication system. The work presented in this paper provides a case study for how current military vehicles' communication systems can be revised to achieve such safe and reliable communication system, in order to enable $\mathrm{DbW}$; and further the work demonstrates how the safety analysis can be used to provide confidence in the system.

Other applications of this research include essentially any system that requires control from a human operator. From flying UAVs to controlling remote turrets, the added capability from a reliable networked control system, complemented by the ability to be controlled from any station, can be suited to tailor any such requirement.

\section{References}

BAE Systems (2016) Terrier ${ }^{\circledR}$ Demonstrating Innovation in Military Vehicles at DSEi. Available online at: www.baesystems.com/en-uk/article/terrierreg-demonstrating-innovation-in-militaryvehicles-at-dsei (accessed on September 2016).

Barbier, E. and Gilhead, P. (2005) DriveWise Hardware Design Process, Ricardo, Inc., Berlin Charter Township, MI.

Charchalakis, P., Valsamakis, G., Stipidis, E., Connor, B. and Ali, F. (2006) 'VSI embedded network integrated systems for military vehicles based on MilCAN', Journal of Defence Science, Vol. 10, No. 4, pp.266-279.

Clay, S. (2011) The Case Against the New Army Ground Combat Vehicle, Cable Assembly Supplier. Available online at: http://www.cableassemblysupplier.com/Articles/2011/CAS Military_Clay_2-8-11.htm (accessed on November 2011).

Connor, R. (2009) Vetronics Standards \& Guidelines, Doc. No. QINETIQ/EMEA/TS/CR0702540, Vehicle Systems Integration (VSI) Programme. Available online at: http://vsi.org.uk/ Standards Metrics/SG_2007_Issue3.pdf (accessed on June 2009). 


\section{Emerging X-by-Wire capabilities}

Hodgson, I. and Make-Kail, M. (2009) Reaping the Benefits of X-by-Wire for Military Land Vehicles, White Paper, Esterel Technologies and TTTech Computertechnik.

iCasualties.org (2016) Iraq and Afghanistan Coalition Military Fatalities. Available online at: http://icasualties.org/ (accessed on September 2016).

Melentis, J., Stipidis, E., Charchalakis, P. and Ali, F. (2008) 'Towards a unified X-by-Wire solution with HUMS, HM \& TTP: lessons learned in implementing it to a Drive-by-Wire vehicle', Proceedings of the IEEE Real Time Networked Systems (RTNS), Rennes, France.

Mitchell, B. and Scutro, A. (2011) SF Deaths Come amid MRAP Rollover Concerns, Army Times. Available online at: http://www.armytimes.com/news/2008/07/marines_MRAP_070108w/ (accessed on November 2011).

Mushenski, C.B., Cartwright, A. and Rydel, S. (2003) Advanced Drive by Wire Design, the Military Solution, SAE Technical Paper, 2003-01-0115.

Nolte, T., Hansson, H. and Bello, L. (2005) 'Automotive communications - past, present and future', 10th IEEE Conference on Emerging Technologies and Factory Automation (ETFA'05), Vol. 1, pp.985-992.

Philippi, S. (2002) 'Modelling and simulation of safety-critical automotive systems', 2002 IEEE International Conference on Systems, Man and Cybernetics, 6-9 October, IEEE, Yasmine Hammamet, Tunisia, p.6.

Pimentel, J. and Sacristan, T. (2001) 'A fault management protocol for TTP/C', The 27th Annual Conference of the IEEE Industrial Electronics Society, IECON'01, IEEE, Denver, CO, USA.

Ricardo AutoFMEA (2011) Available online at: http://www.ricardo-autofmea.com (accessed on November 2011).

Ruhlen, B. (2009) 'Presentation of MRAP IED effects' (Major Ruhlen Brent, Senior Project Officer, TCM-BC, CP Division), Pictures released into the public domain as Unclassified, Fort Leavenworth Kansas, USA.

Summers, D., Charchalakis, P., Stipidis, E. and Ali, F. (2006) 'FlexRay-MilCAN bridging', Vehicle Power and Propulsion Conference (VPPC'06), IEEE, Windsor, UK.

Summers, D., Melentis, J., Charchalakis, P., Stipidis, E. and Ali, F. (2009) 'Through life capability vetronics systems integration testbed', Codex Unclassified Journal of the Ministry of Defence, No. 3, pp.68-79.

TTTech Computertechnik (2007) Safety Manual for TTC 200, Document Edition 1.0.8 of 14th November 2007, TTTech Computertechnik, Vienna.

TTTech Computertechnik (2011) TTP Tools, Design and Development Software, TTTech Computertechnik, Vienna. Available online at: http://www.tttech.com/products/ttp/designdevelopment-software/ (accessed on November 2011).

UK MoD (2010) Generic Vehicle Architecture, DEFSTAN23-09, No. 1, 20 August, UK.

US DoD (1980) Procedures for Performing a Failure Mode Effect and Criticality Analysis, MILSTD-1629A, US Department of Defense, Alexandria, VA.

VRC (2012) Mobile Platform Demonstrator ('Buggy'), Vetronics Research Centre. Available online at: http://www.vetronics.org/research/buggy/ (accessed on January 2012).

X-by-Wire Project (1998) Safety Related Fault Tolerant Systems in Vehicles, Final Report, Brite Euram, Rep. No. X-by-Wire-DB-6-6/6-24, Stuttgart, Germany. 